substances for medical and scientific purposes is indispensable and that their availability for such purposes should not be unduly restricted ...."

In contrast, part of Article 7 states that in respect of substances in Schedule I, the parties shall: " $(a)$ prohibit all use except for scientific and very limited medical purposes by duly authorized persons, in medical or scientific establishments which are directly under the control of their Governments or specifically approved by them; (b) require that manufacture, trade, distribution, and possession be under a special licence or prior authorization; (c) provide for close supervision of the activities and acts mentioned in paragraphs $(a)$ and (b); (d) restrict the amount supplied to a duly authorized person to the quantity required for his authorized purpose; $(e)$ require that persons performing medical or scientific functions keep records concerning the acquisition of the substances and the details of their use, such records to be preserved for at least two years after the last use recorded therein ...."

When a country ratifies this new convention the responsibility for control and all of the legal and administrative interpretations will undoubtedly be vested in the national government. Moreover-if history is any guide-this power and authority will be given to governmental officials, whose prime interest is in control of the drugs-with relatively low priority given to science. For example, our sad experience with cannabis, whereby a tremendous hedge of legal and administrative controls virtually precluded the development of bona fide scientific investigations, is a classic example of an unbalanced system that should never again be allowed to develop. Admitting the need for appropriate measures of control through the national government, the major policy questions that must be clarified are: Does the government by virtue of its responsibility for the control of drugs also retain control of scientific investigations that require supplies of the controlled drugs for research purposes? Who is going to determine the kinds of research and the priorities-the control officials or the scientists?

An appropriate answer to these questions is crucial to the development of responsible scientific work. Moreover, in the case of the United Kingdom they should be debated and settled before the new international convention is ratified by Parliament. In my opinion, the determination of research priorities and investigations should be vested in the appropriate scientific body, not in the administrative officials.-I am, etc.,

H. David ARchibald Executive Director,
Research Foundation Toronto, Canada

1 United Nations, Conference for the Adoption of a Protocol on Psychotropic Substances, E/Conf.
58/6, February 1971.

\section{Camphorated Oil}

SIR,-We feel that the "over the counter" purchase of drugs on the market gives easy access to some very dangerous pharmaceutical preparations. Recently a man was admitted to the Regional Poisons Unit following an attempted suicide with an overdose of $150 \mathrm{ml}$ of camphorated oil (B.P.
$20 \%$ ). The potential lethal dose in an adult is $20 \mathrm{ml}$. He was in peripheral circulatory shock and severely dehydrated owing to vomiting. After immediate resuscitation gastric lavage was carried out under general anaesthesia using a cuffed endotracheal tube. Soon after admission he developed three attacks of severe and prolonged grand mal, which subsided with intravenous diazepam. Following intensive supportive treatment he was fit and well 36 hours later. To the best of our knowledge this is one of the highest doses to be survived.

Camphorated oil has in the past been used for the purpose of abortion because the substance crosses the placenta. It causes death due to respiratory depression. There is no specific antidote. However, haemodialysis utilizing soyabean oil has been employed in the past.

In this day and age camphorated oil has lost its former medical indications. Its danger as a poison by far outweighs its usefulness, if any, and we do not think that camphorated oil now has a place in the open market.-We are, etc.

\section{S. J. KaRAYANNOPOULOS}

Department of Medicine,

Royal Infirmary, Sunderland

\section{Pharmacologically Active I.U.D.s}

SIR,-At a time when concern at the increase in iatrogenic disease is both opportune and fashionable I should like to draw attention to and deplore a recent new development in contraception. This is experimentation with pharmacologically active intrauterine devices, notably those wound with metallic copper wire.

The rationale behind their development is that, whereas for inactive devices pregnancy rates appear to be roughly proportional to surface area and large devices carry compensating disadvantages, copper ions have a directly contraceptive effect, so that devices can be kept small to minimize other inconveniences, leaving the copper to take care of the risks of pregnancy.

Where a major gain in efficacy is at stake clearly we must be ready to accept a temporary increase in uncertainty regarding the safety of a new device or drug. No progress is possible otherwise. In this instance, however, this is not the case. The more advanced of the inert I.U.D.s (the Dalcon shield, for example) have, if the results of trials so far available are a guide, rates of pregnancy, expulsion, and medical sideeffects low enough so that no improvements could be classified as major advances. Improvements should of course be sought continually, but there must be a limit on their price. Pharmacologically active I.U.D.s introduce a new dimension of unknown longterm hazards, and I suggest that this is too high a price for the small gains remaining to be made.

We know nothing of the properties of the endometrium as a route for medication nor of the effects of continuous exposure to a weak solution of copper salts on it, on myometrium or cervix. We have no experience of intermittent massage with such a solution of vagina, glans penis, and urethral meatus. We shall have to await with uncertainty the outcome of pregnancies that will take place with the device in situ and to speculate on the performance of a possibly damaged myometrium during labour even in pregnancies occurring after its removal. Allergic reactions, as with the topical use of copper salts elsewhere, are to be expected. An added source of anxiety regarding carcinogenesis, this time in both male and female genital tracts, will have to be studied for 20 years or more.

In addition, it should be recognized that the advantages of the active I.U.D.s are fairy gold. With present copper devices, after a relatively short period the active substance becomes exhausted or inactivated, and removal and reinsertion is necessary. However, I understand that promising work is in progress to extend the life time of the device. Until this has been achieved the application of copper I.U.D.s to mass programmes in developing countries is likely to give rise after a short period to unacceptably high pregnancy rates. The difficulties and costs of attempting to arrange for regular renewal of all I.U.D.s in such settings are virtually insurmountable.

The introduction of pharmacologically active I.UD.s is gratuitous furor medicandi. I suggest we should continue to have confidence in the steady progress being made towards the perfection of inert devices and have nothing to do with this complex, hazardous, and unnecessary new industry.-I am, etc.

Population Bureau, London S.W.1

D. WOLFERS

\section{Phenformin Overdose}

SIR,-Phenformin is an oral hypoglycaemic agent related to the biguanide group and is in increasing usage. In $1969 \quad 156,000$ National Health prescriotions for phenformin were made. ${ }^{1}$ Combined with an oestrogen it has recently been used experimentally in fibrinolytic therapy. ${ }^{2}$ One of the presentations of phenformin is as a $50 \mathrm{mg}$ slow-release capsule and the therapeutic range is up to 3 capsules daily.

On 31 August a white male aged 30 years presented with collabse. having vomited about a pint and a half $(800 \mathrm{ml})$ of altered blood, with fresh blood, during the evening and morning before admission. When examined he was in peripheral circulatory failure, but apart from some midline epigastric tenderness and fresh melaena stool in the rectum there were no physical signs.

During a medical examination for a job abroad 10 months before he was found to have glycosuria. Subsequent blood sugars were raised above normal and he was given soluble insulin. His insulin requirements were low and he was changed to phenformin $50 \mathrm{mg}$ slow-release capsules and a $200 \mathrm{~g}$ carbohydrate diet.

Throughout the 6 months before admission he took his capsules according to his urine testing with Clinitest tablets. This

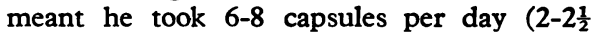
times the maximum effective therapeutic dose). He lost 2 stone $(12.7 \mathrm{~kg})$ in weight in the four months before admission, and had anorexia with central low epigastric discomfort but no symptoms of gastric reflux. He dated the onset of symptoms from when he started phenformin. There was no previous history of peptic ulcer nor a family history.

He was transfused a total of four units of whole blood and treated medically with antacids, antiemetics, and analgesics. A 